\section{RMD Open}

Rheumatic \&

Musculoskeletal Diseases

\title{
Effect of celastrol on bone structure and mechanics in arthritic rats
}

\author{
Rita Cascão, ${ }^{1}$ Bruno Vidal, ${ }^{1}$ Mikko Arttu Jalmari Finnilä, ${ }^{2,3}$ \\ Inês Pascoal Lopes, ${ }^{1}$ Rui Lourenço Teixeira, ${ }^{1,4}$ Simo Saarakkala, ${ }^{2,5}$ \\ Luis Ferreira Moita, ${ }^{6}$ João Eurico Fonseca ${ }^{1,4}$
}

To cite: Cascão R, Vidal B, Jalmari Finnilä MA, et al. Effect of celastrol on bone structure and mechanics in arthritic rats. RMD Open 2017;3:e000438. doi:10.1136/ rmdopen-2017-000438

- Prepublication history and additional material for this paper are available online. To view please visit the journal (http://dx. doi.org/10.1136/rmdopen-2017 000438).

$\mathrm{RC}$ and BV contributed equally.

LFM and JEF are joint senior authors.

Received 18 January 2017 Revised 17 July 2017

Accepted 28 July 2017

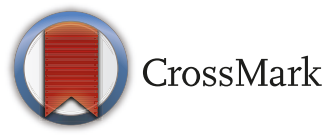

${ }^{1}$ Instituto de Medicina Molecular, Faculdade de Medicina, Universidade de Lisboa, Lisbon, Portugal

${ }^{2}$ Research Unit of Medical Imaging, Physics and Technology, Faculty of Medicine University of Oulu, Oulu, Finland ${ }^{3}$ Department of Applied Physics, University of Eastern Finland, Kuopio, Finland

${ }^{4}$ Department of Rheumatology, Centro Hospitalar de Lisboa Norte, EPE, Hospital de Santa Maria, Lisbon Academic Medical Centre, Lisbon, Portugal ${ }^{5}$ Department of Diagnostic Radiology, Oulu University Hospital, Oulu, Finland ${ }^{6}$ Instituto Gulbenkian de Ciência, Oeiras, Portugal

Correspondence to Dr Rita Cascão;

ritacascao@medicina.ulisboa.pt

\section{ABSTRACT}

Objective Rheumatoid arthritis (RA) is characterised by chronic inflammation leading to articular bone and cartilage damage. Despite recent progress in RA management, adverse effects, lack of efficacy and economic barriers to treatment access still limit therapeutic success. Therefore, safer and less expensive treatments that control inflammation and bone resorption are needed. We have previously shown that celastrol is a candidate for RA treatment. We have observed that it inhibits both interleukin (IL)-1 $\beta$ and tumor necrosis factor (TNF) in vitro, and that it has anti-inflammatory properties and ability to decrease synovial CD68+ macrophages in vivo. Herein our goal was to evaluate the effect of celastrol in local and systemic bone loss.

Methods Celastrol was administrated intraperitoneally at a dose of $1 \mu \mathrm{g} / \mathrm{g} /$ day to female Wistar adjuvantinduced arthritic rats. Rats were sacrificed after 22 days of disease progression, and blood, femurs, tibiae and paw samples were collected for bone remodelling markers quantification, 3-point bending test, micro-CT analysis, nanoindentation and Fourier transform infrared spectroscopy measurements, and immunohistochemical evaluation.

Results We have observed that celastrol preserved articular structures and decreased the number of osteoclasts and osteoblasts present in arthritic joints. Moreover, celastrol reduced tartrate-resistant acid phosphatase $5 \mathrm{~b}$, procollagen type 1 amino-terminal propeptide and $\mathrm{C}$ terminal crosslinked telopeptide of type II collagen serum levels. Importantly, celastrol prevented bone loss and bone microarchitecture degradation. Celastrol also preserved bone nanoproperties and mineral content. Additionally, animals treated with celastrol had less fragile bones, as depicted by an increase in maximum load and yield displacement.

Conclusions These results suggest that celastrol reduces both bone resorption and cartilage degradation, and preserves bone structural properties.

\section{INTRODUCTION}

Rheumatoid arthritis (RA) is a chronic immune-mediated inflammatory disease with an estimated worldwide prevalence of $1 \%$. RA is characterised by chronic oedema and inflammation of the synovial tissue that lines the joints. As disease progresses, cartilage

\section{Key messages}

What is already known about this subject?

- Celastrol has anti-inflammatory properties.

What does this study add?

- Celastrol reduces bone resorption and cartilage degradation.

- Celastrol preserves bone structure.

How might this impact on clinical practice?

- Based on these data on animal models Celastrol may serve as a useful therapeutic agent for rheumatoid arthritis.

and bone are damaged, leading to articular destruction. ${ }^{1}$ This periarticular and systemic bone loss is responsible for an increased risk of fracture in patients. ${ }^{2-5}$ Bone loss in RA results from an imbalance between the osteoblastic synthesis and osteoclastic degradation of bone, with bone resorption dominating over bone formation. ${ }^{6}$

We have previously reported increased levels of interleukin (IL)-1 $\beta$ in very recent onset arthritis and in the synovial fluid of patients with established RA. ${ }^{7}$ This may be explained by the activation of caspase- 1 , responsible for the processing of pro-IL-1 $\beta$, which we have also observed to be increased since early RA. ${ }^{8}$ Through an in vitro drug screening using the THP-1 macrophage-like cell line, we have identified compounds that decrease the production of IL-1 $\beta$ and tumor necrosis factor (TNF), another central proinflammatory cytokine of RA physiopathology. Among them, celastrol was identified as a promising therapeutic candidate for arthritis due to its ability to downregulate the production of IL-1 $\beta$ and TNF, by inhibiting both the activation of caspase- 1 and nuclear factor kappa $B$ $(\mathrm{NF}-\mathrm{kB}) .{ }^{9}$ Celastrol is a pentacyclic-triterpene extracted from a plant used in traditional Chinese medicine, the Tripterygium wilfordii Hook $F$ (TwHF). The anti-inflammatory 
properties of celastrol have been mainly attributed to the regulation of cytokine and chemokine production and inflammatory mediators expression, ${ }^{10-14}$ the modulation of inflammatory cell functions, ${ }^{15-18}$ and the in vitro regulation of osteoclasts. ${ }^{19}{ }^{20}$ We have recently described in vivo that celastrol has significant anti-inflammatory and antiproliferative properties, with a decrease in the overall synovial inflammatory cellularity, and most importantly in the number of sublining CD68+ macrophages, a biomarker of drug efficacy in RA. ${ }^{9}{ }^{21}$ In this study, we have hypothesised that celastrol is able to control inflammation, and the focal and systemic bone resorption that occurs in arthritis.

Our aim was to evaluate the ability of celastrol to prevent disturbances on bone structure and strength induced by inflammation in the adjuvant-induced arthritis (AIA) rat model.

\section{MATERIALS AND METHODS Animals}

Eight-week-old female Wistar AIA rats weighing 230-250 g were purchased from Charles River Laboratories International (Wilmington, USA). Charles River Laboratories performed optimised induction of adjuvant disease, using Freund's complete supplemented with mycobacterium and injected in the right footpad (solution: $40 \mathrm{mg}$ of mycobacterium $/ 1 \mathrm{~mL}$ of paraffin oil; $0.1 \mathrm{~mL}$ of the solution per animal). AIA rats were maintained in specific pathogen-free facilities, randomly housed per groups under standard laboratory conditions $\left(\right.$ at $20^{\circ} \mathrm{C}-$ $22^{\circ} \mathrm{C}$ under 10-hour light/14-hour dark), and given free access to food (RM3, SDS Diets, Witham, UK) and water (Ultrapure). In addition, to minimise animal discomfort paper shavings were used as bedding material in Double Decker GR1800 cages (Tecniplast, Kettering, UK) with five animals per cage. In accordance with Directive 2010/63/EU, all animal procedures were approved by the institutional animal welfare body (Órgão Responsável pelo Bem-estar Animal, ORBEA-iMM) and licensed by the Portuguese competent authority (Direcção Geral de Alimentação e Veterinária, licence number: 0421/000/000/2016). Humane end-points were established and animals were sacrificed when presenting the maximum inflammatory score $(0-3)^{22}$ in more than two paws or when presenting more than $20 \%$ of body weight loss.

\section{Celastrol treatment}

The dose of celastrol $(1 \mu \mathrm{g} / \mathrm{g} /$ day $)$ used in this study was based on that used in our previous studies ${ }^{921}$ and in other studies. ${ }^{23}$ Also, we have already reported that this dose is effective in suppressing synovial inflammation in the AIA rat model, with no evidence of drug-induced toxicity. ${ }^{21}$ Celastrol (Sigma, Sintra, Portugal) stock solution of $100 \mathrm{mg} / \mathrm{mL}$ in dimethyl sulfoxide (DMSO) was dissolved in normal saline solution and injected intraperitoneally in the early treatment group of AIA rats since the fourth day of disease induction $(\mathrm{n}=15)$ and in the late treatment group since the 11th day of disease induction $(n=15)$, and was maintained until day 22. Studies using the acute AIA model are generally completed at this time point due to a plateau effect of inflammatory manifestations. A group of healthy non-arthritic and arthritic untreated female age-matched Wistar rats sacrificed on day 22 after disease induction (chronic clinical stage) were used as controls in all experiments. The sample size in each group was calculated using free sample size calculating $\mathrm{G}^{*}$ Power V.3.1.9.2 software (type of power analysis: a priori; alpha $(\alpha)$ error probability: 0.05; power (1-beta $(\beta)$ error probability): 0.95; effect size d: 1.526112; actual power: 0.9576654$)$. At the preclinical AIA progression stage, evidence of inflammation or bone erosions is still lacking in the contralateral hind paw and fore paws. Hind paw swelling, inflammation and joint erosions steadily progress during acute clinical stage and reach a plateau in the chronic stage. ${ }^{24}$ Rats were sacrificed by $\mathrm{CO}_{2}$ narcosis, and blood, femurs, tibiae and paw samples were collected.

\section{Immunohistochemical staining of cathepsin $\mathrm{K}$ and osteocalcin positive cells in hind paws}

Left hind paw samples, from the ankle joint, collected at the time of sacrifice were fixed immediately in $10 \%$ neutral buffered formalin solution and then decalcified in $10 \%$ formic acid. Samples were next dehydrated and embedded in paraffin, serially sectioned at a thickness of $5 \mu \mathrm{m}$ using a microtome, and mounted on microscope slides. Osteoclasts and osteoblasts immunolocalisation in subchondral bone tissue at the tibia/talus region was performed by staining with cathepsin $\mathrm{K}$ (osteoclast marker; mature osteoclast enzyme; Biorbyt, Cambridge, UK) and osteocalcin (osteoblast marker; indicator of osteoblastic activity; Abcam, Cambridge, UK) primary antibodies followed by EnVision+ (Dako, Glostrup, Denmark). Colour was developed in solution containing diaminobenzidine-tetrahydrochloride (Sigma, Sintra, Portugal), $0.5 \% \quad \mathrm{H}_{2} \mathrm{O}_{2}$ in phosphate-buffered saline buffer ( $\mathrm{pH}$ 7.6). Slides were counterstained with haematoxylin and mounted. Immunohistochemical evaluation of rat joints was performed in a blinded fashion using a semiquantitative score of 0-3 (0: 0\%-25\% staining; 1 : 25\%-50\% staining; 2: $50 \%-75 \%$ staining; 3 : more than $75 \%$ staining) of the positively stained cells per total cellular count in the tissue section. ${ }^{9}{ }^{21}{ }^{25}$ Slides were analysed using a Leica DM2500 microscope (Leica Microsystems, Wetzlar, Germany).

\section{Serum biochemical measurement of bone and cartilage turnover markers}

Bone and cartilage turnover was analysed by quantifying the levels of TRACP-5b (tartrate-resistant acid phosphatase 5b), P1NP (procollagen type 1 amino-terminal propeptide) and CTX-II (C terminal crosslinked telopeptide of type II collagen) in rat serum using ELISA (Immunodiagnostic Systems, Boldon, UK). All the commercial assays were performed according to the manufacturers' 
instructions, and standard curves were generated using supplied reference concentrations. Samples were measured using a plate reader Infinite M200 (Tecan, Mannedorf, Switzerland).

\section{Micro-CT analysis}

Structural properties of the trabecular and cortical tibiae were determined with a high-resolution micro-CT system (SkyScan 1272, Bruker micro-CT, Kontich, Belgium). Moist bones were wrapped in parafilm and covered with dental wax to prevent drying and movement during the scanning. X-ray tube voltage was set to $50 \mathrm{kV}$ and the beam was filtered with $0.5 \mathrm{~mm}$ aluminium filter. Sample position and camera settings were tuned to provide $3.0 \mu \mathrm{m}$ isotropic pixel size and projection images were collected every $0.2^{\circ}$. Density calibration was performed against hydroxyapatite phantoms with densities of $250 \mathrm{mg} / \mathrm{cm}^{3}$ and $750 \mathrm{mg} / \mathrm{cm}^{3}$. Image reconstruction was done with NRecon software (V.1.6.9.8; Bruker micro-CT) and appropriate corrections were applied to reduce beam hardening and ring artefacts. Trabecular bone was manually segmented from cortical bone, and trabecular bone parameters were analysed over 1400 slices, starting 200 slices distal from growth plate. Cortical bone parameters were analysed over 300 slices, starting 1800 slices distal from growth plate. Analyses were performed in agreement with guidelines for assessment of bone microstructure in rodents using micro-CT. ${ }^{26}$ Trabecular bone morphology was analysed by applying global threshold and despeckling to provide binary image for three-dimensional (3D) analyses. Cortical bone region of interest (ROI) was refined with ROI-shrink wrap operation, which also provided cortical bone shape for two-dimensional morphological analysis. This was followed by segmentation of blood vessels using adaptive thresholding. Blood vessels (porosity) were analysed using 3D morphological analyses.

\section{Nanoindentation measurements}

Nanoindentation was performed using a CSM-Nano Hardness Tester System (Indentation V.3.83; CSM Instruments SA, Switzerland) equipped with a Berkovich-based pyramid diamond indenter. After micro-CT, $0.5 \mathrm{~mm}$ of top tibia was cut and proximal part was embedded to low viscosity epoxy resin (EpoThin, Buehler, Knorring $\mathrm{Oy} \mathrm{Ab}$, Helsinki, Finland). Slow-speed diamond saw was used to remove $10 \%$ of bone length. The sample surface was polished using silicon carbide sandpaper with a decreasing grid size $(800,1200,2400$ and 4800) and finished with cloth containing $0.05 \mu \mathrm{m}$-alumina particles. Indentation protocol was adopted from previous work, ${ }^{27}$ and an average of eight indentations were done on both cortical and trabecular bone with quasistatic (so called 'advanced') loading protocol. All indentations were performed under an optical microscope to achieve the precise location of indentations at the centre of the targeted area in the tissue. ${ }^{28}$ In the advanced protocol, a trapezoidal loading waveform was applied with a loading/ unloading rate of $20 \mathrm{mN} / \mathrm{min}$, and with an intermediate load-hold-phase lasting $30 \mathrm{~s}$ hold at a maximum load of $10 \mathrm{mN}$. The hardness (HIT), indentation modulus (EIT), indentation creep (CIT) and elastic part of indentation work $(\eta \mathrm{IT})$ were measured by advanced protocol using the Oliver and Pharr $^{29}$ method. Histological images of rat tibiae from diaphyseal cortical region were acquired during the nanoindentation technique, using a CSM Instruments microscope equipped with a colour camera. A histological score was applied to evaluate the lamellar structures of bone tissue. This evaluation was performed in a blind fashion using a semiquantitative score for the analysis of the lamellar bone structure (1: predominantly parallel-lamellae; 2: concentric and parallel-lamellae in the same proportion; 3: predominantly concentric lamella). The ratio of osteocyte lacuna area/ total tissue area was also evaluated at $\times 200$ magnification to analyse the percentage of total tissue area occupied by osteocyte lacunae. The method of acquisition and analysis used was the same applied for the evaluation of bone volume/tissue volume in histomorphometry technique. ${ }^{30}$ All variables were expressed and calculated according to the recommendations of the American Society for Bone and Mineral Research, ${ }^{31}$ using a morphometric program (Image J 1.46R with plugin BoneJ).

\section{FTIR quantification}

Samples used for nanoindentation were also used for Fourier transform infrared spectroscopy (FTIR). Chemical composition was measured from bone surfaces separately with the HYPERION 3000 FTIR microscope (Bruker Optics, Billerica, Massachusetts, USA) using attenuated total reflection (ATR) objective. The ATR crystal was compressed on the bone with a constant load, and spectral images were recorded with a focal plane array detector. Spatial and spectral resolutions were set to $1 \mu \mathrm{m}$ and $2 \mathrm{~cm}^{-1}$, respectively. Each spectrum between 840 and $3300 \mathrm{~cm}^{-1}$ was averaged 32 times, and two spectral maps $(32 \times 32$ spectra) were collected from the trabecular and cortical bone separately. Data were analysed using a custom script in the Matlab environment (MathWorks, Natick, Massachusetts, USA). For each spectral map, areas under curves were calculated for amide I, phosphate and carbonate peaks by integrating spectra between 1595$1720 \mathrm{~cm}^{-1}, 900-1185 \mathrm{~cm}^{-1}$ and $850-895 \mathrm{~cm}^{-1}$, respectively. Blood vessels and other porous structures were removed by excluding spectra with maximum phosphate peak height less than 0.5 absorbance units. Average content as well as well-established parameters for bone composition (carbonate:amide I, mineral:matrix and carbonate:phosphate) were finally calculated from the threshold spectral maps. $^{32}$

\section{3-point bending biomechanical test}

In order to investigate bone strength after celastrol treatment, femurs were subjected to a 3-point bending test in a universal testing machine (Instron 3366, Instron, Norwood, USA) with a load-cell of 500 N. Femurs were 
placed horizontally, anterior side upwards, on a support with span length of $5 \mathrm{~mm}$. The load was applied with a constant speed of $0.005 \mathrm{~mm} / \mathrm{s}$ until a failure occurred. Stiffness was analysed by fitting first-degree polynomial function to the linear part of recorded load deformation data. The breaking point was defined when force reached a maximum value, and corresponding deformation and absorbed energy were analysed.

\section{Statistical analysis}

The primary outcome of this study was to prevent the structural and mechanical damage of bone induced by inflammation, and the secondary outcome was to treat the structural and mechanical deterioration of bone in a chronic phase of arthritis development in the AIA rat model.

A number of characteristics, described above, were analysed using descriptive statistics (median with minimum and maximum interval for continuous variables) and box-plots.

Differences between the three independent groups (celastrol early and late treatment groups, and untreated arthritic rats) on these characteristics were assessed by non-parametric Mann-Whitney tests with Bonferroni correction. Thus, applying the Bonferroni correction, we divided the global significance level at 0.05 by the number of independent tests (3) to get the Bonferroni critical value, so a test would have to have $p$ value $<0.02$ to be significant.
The Bonferroni correction is the most conservative correction. However, it is mainly useful when there are a fairly small number of multiple comparisons and we are looking for one or two that might be significant, as is here the case.

The Mann-Whitney test was also used to compare differences between the other two independent groups: healthy non-arthritic and untreated arthritic rats on the dependent variables. In these cases, $p$ values $<0.05$ were considered to be significant.

All inferential statistical analyses were performed using the GraphPad Prism V.5.01 (GraphPad, La Jolla, USA).

\section{RESULTS}

\section{Celastrol effect on bone-related cells in arthritic joints}

At treatment baseline, celastrol early-treated group had a mean inflammatory score of $1.5 \pm 0.7$ and celastrol latetreated group had a mean inflammatory score of 3.9 \pm 2.0 . By the end of the treatment, both celastrol early-treated $(0.7 \pm 0.9)$ and late-treated $(1.5 \pm 0.2)$ groups reduced the inflammatory score compared with arthritic animals $(8.0 \pm 2.0)$, with no differences in body weight. ${ }^{21}$

Arthritic rats showed an increase in the number of osteoclasts (cathepsin $\mathrm{K}+$ cells) compared with healthy controls (figure 1). Importantly, celastrol administration significantly lowered the number of osteoclasts in comparison to arthritic rats. Arthritic rats also showed an increase in the number of osteoblasts (osteocalcin
A
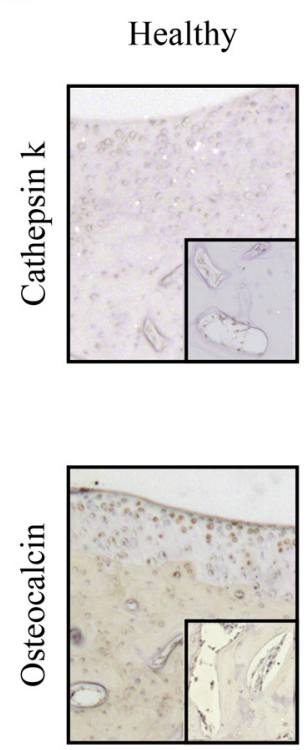

Arthritic
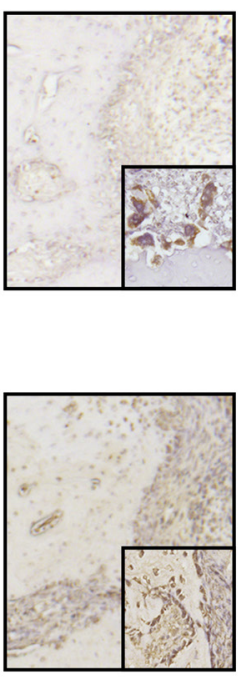

Celastrol early group
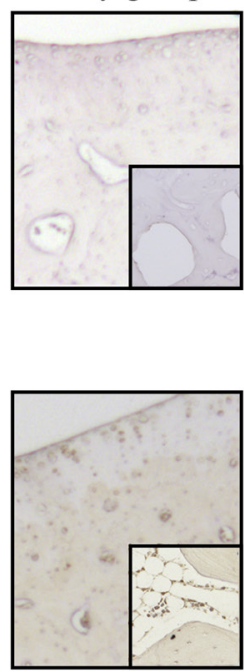

Celastrol late group
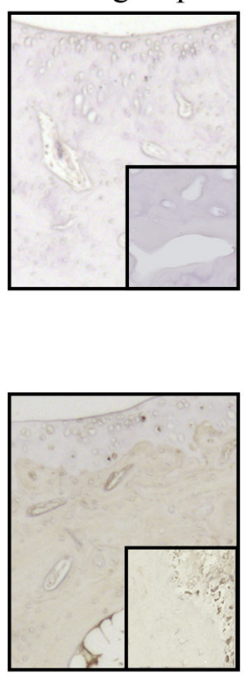

B
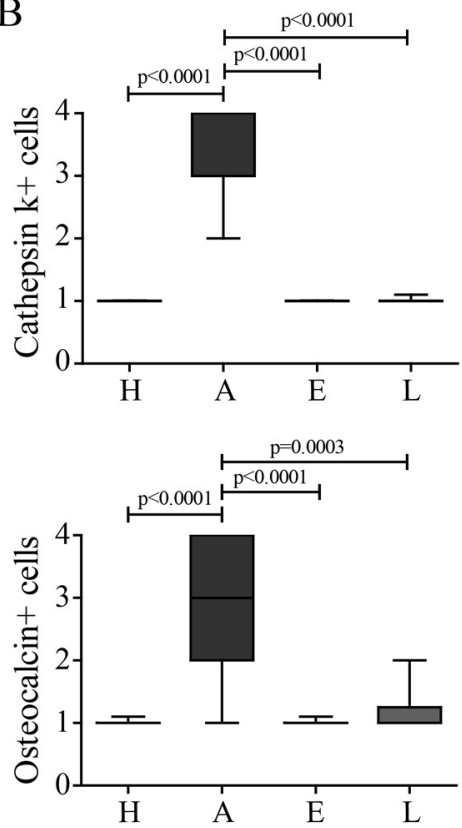

Figure 1 Celastrol reduces the number of bone-related cells in arthritic joints. (A) Representation of the immunohistochemical evaluation performed in left hind paw sections at the ankle joint by day 22 after celastrol treatment. Magnifications of $200 \times$ and 400x. (B) Cathepsin K+ cells and osteocalcin positive cells were identified by immunohistochemistry of paw sections. Immunohistochemical analysis was performed using a semiquantitative score. Notice that celastrol treatment significantly reduced both types of cells. Samples were collected at the time of sacrifice. Data are expressed as median score with minimum and maximum interval. $\mathrm{H}$, healthy; $\mathrm{A}$, arthritic; $\mathrm{E}$, celastrol early-treated; L, celastrol late-treated. Healthy, $\mathrm{n}=16$; arthritic, $n=10$; celastrol early-treated, $n=15$; and celastrol late-treated, $n=15$. 

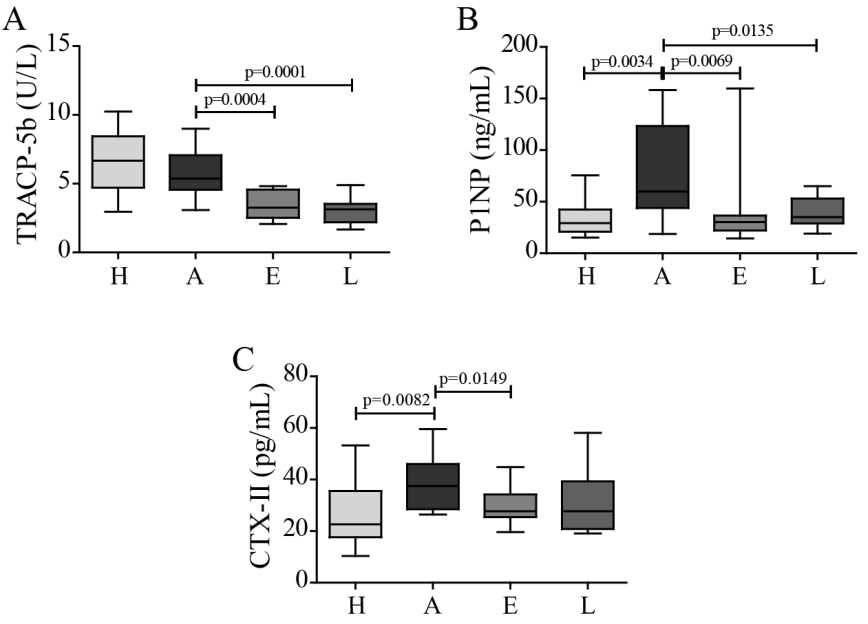

Figure 2 Celastrol diminishes bone and cartilage resorption markers. (A) TRACP-5b, (B) P1NP and (C) CTX-Il levels were quantified in rat serum samples collected at the time of sacrifice. Celastrol is able to significantly reduce the levels of TRACP-5b, P1NP and CTX-II in comparison with untreated arthritic rats. Data are expressed as median with minimum and maximum interval. $\mathrm{H}$, healthy; $\mathrm{A}$, arthritic; $\mathrm{E}$, celastrol early-treated; $L$, celastrol late-treated. Healthy, $n=13$; arthritic, $n=18$; celastrol early-treated, $n=15$; and celastrol late-treated, $n=15$. CTX-II, $C$ terminal crosslinked telopeptide of type II collagen; P1NP, procollagen type 1 amino-terminal propeptide; TRACP-5b, tartrate-resistant acid phosphatase $5 b$.

positive cells lining bone surfaces) compared with healthy controls (figure 1). Notably, celastrol administration significantly reduced the number of these cells in comparison to arthritic animals.

\section{Celastrol effect on bone resorption and cartilage degradation}

To further elucidate the protective effect of celastrol on inflammation-mediated joint damage, bone and cartilage degradation markers were quantified. We measured serum levels of TRACP-5b as a marker of bone resorption, P1NP as a marker of bone formation and CTX-II as a maker of cartilage degradation. In the arthritic group, there was no significant increase in TRACP-5b levels at the end of the study period (figure 2A). TRACP-5b increases immediately after disease onset, and then gradually decreases throughout arthritis progression (online supplementary figure S1), as previously described by Stolina et al. ${ }^{33}{ }^{34}$ Importantly, both in celastrol earlytreated and late-treated rats, there was a significant reduction in TRACP-5b levels compared with arthritic rats, suggesting a decrease in bone resorption. This reduction was also noticed in both treatment groups at an earlier time point ( $\mathrm{p}=0.0014$ in celastrol early-treated vs arthritic rats at baseline, day 4; and $\mathrm{p}<0.0001$ in celastrol latetreated vs arthritic rats at baseline, day 11; online supplementary figure $\mathrm{S} 1$ ). In arthritic rats, there was an increase in serum P1NP (figure 2B) as a consequence of the high bone turnover, and in both treatment groups there was a significant drop in these levels compared with arthritic group. Finally, arthritic rats also revealed an increase in
CTX-II levels due to cartilage degradation (figure 2C). Celastrol treatment significantly decreased these levels revealing that it is also effective in protecting cartilage integrity. Of note, although a strong tendency towards a decrease in CTX-II was observed in celastrol late-treated rats, it did not reach statistical significance.

\section{Celastrol effect on bone structure}

The effect of celastrol on inflammation-induced systemic bone loss was assessed by micro-CT analysis of tibial bones (figure 3). Representative reconstructions of micro-CT analysis of rat tibiae are shown in figure 3A. Arthritis progression led to significant reductions in trabecular bone mass and in trabecular bone volume fraction and number, and an increase in trabecular separation and porosity, compared with healthy controls (figure 3B). Also, the structural integrity declined with arthritis, as trabeculae had fewer connections and had rather rod-like appearance, indicated by increased structure model index. An 18-day course of therapy with celastrol, starting 4 days after disease induction, preserved bone mass and integrity, with a significant increase in trabecular bone volume fraction $(+16.6 \%)$ and number $(+20.3 \%)$, as well as with a significant decrease in trabecular separation $(-12.9 \%)$ and porosity $(-4.5 \%)$, in comparison to arthritic rats. Celastrol treatment also preserved structural integrity, as trabeculae have more connections and have less rod-like appearance.

A similar pattern was observed in cortical bone (figure 3C). Arthritis decreased cortical bone area $(-10.8 \%, \mathrm{p}<0.0001)$ and thickness $(-11 \%, \mathrm{p}<0.0001)$ compared with healthy controls. Although overall cortical porosity was similar between arthritic and healthy controls, blood vessel channels were wider in arthritic bones. Both treatment approaches affected cortical bone by inhibiting bone resorption as shown by significantly smaller endosteal volume $(-14.5 \%$ in early-treated and $-20.1 \%$ in late-treated rats vs arthritic animals). Also both groups have significantly decreased cortical porosity $(-18.2 \%$ in early-treated and $-30.1 \%$ in late-treated rats $)$ due to a decrease in the number $(-17.1 \%$ in early-treated and $-29.7 \%$ in late-treated animals) and thickness $(-3.1 \%$ in early-treated and $-4.8 \%$ in late-treated rats) of blood vessel channels, and thus significantly increasing their separation $(+7.9 \%$ in early-treated and $+14.6 \%$ in latetreated groups) compared with arthritic rats.

\section{Celastrol effect on bone matrix quality}

Nanoindentation was performed in order to assess the quality at tissue matrix level (figure 4). Nanomechanical tests revealed that arthritic rats had a decrease in the hardness of cortical and trabecular bone in comparison with healthy controls (figure 4A). In contrast, rats treated with celastrol showed an increase in cortical bone hardness; however, the same was not observed in trabecular bone. In addition, topographic images gathered during nanoindentation allowed the characterisation of bone histological features (figure 4B). Concentric lamellae 
A

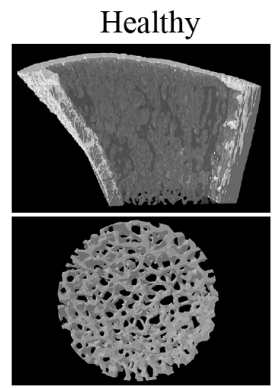

$\mathrm{B}$

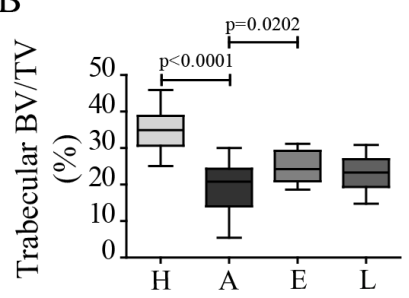

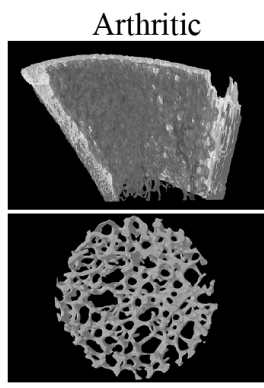

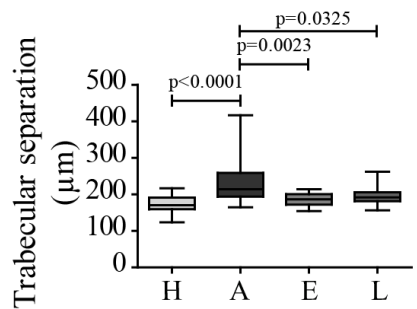

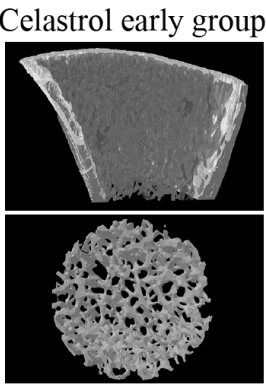

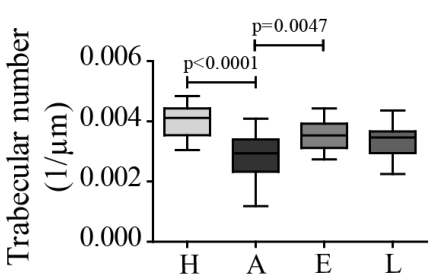

Celastrol late group
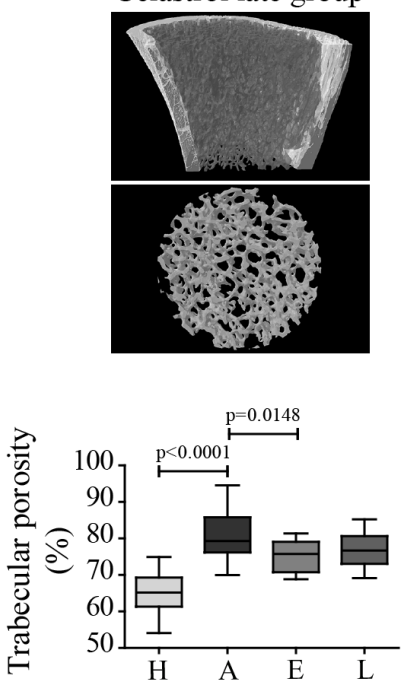

$\mathrm{C}$
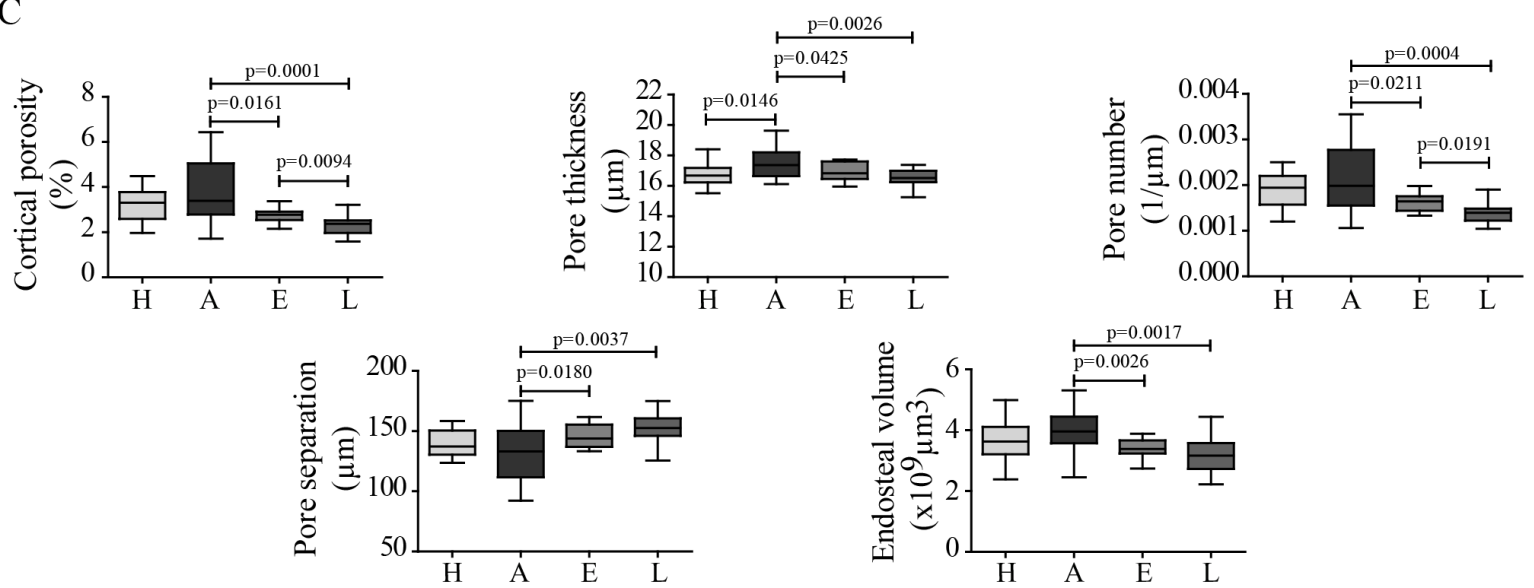

Figure 3 Celastrol preserves bone microarchitecture in arthritis. Inflammation-induced bone loss and bone microarchitecture degradation and the protective effect of celastrol are illustrated in representative micro-CT reconstructions (A). Trabecular (B) and cortical (C) bone indices were quantified from micro-CT reconstructions. Notice that tibiae from the celastrol earlytreated group have improved trabecular and cortical parameters compared with arthritic rats. Tibiae were collected at the time of sacrifice. Data are expressed as median with minimum and maximum interval. BV/TV, bone volume per tissue volume; $\mathrm{H}$, healthy; A, arthritic; E, celastrol early-treated; L, celastrol late-treated. Healthy, $n=30$; arthritic, $n=30$; celastrol early-treated, $\mathrm{n}=15$; and celastrol late-treated $\mathrm{n}=15$.

were identified in secondary osteons and were more frequently observed in arthritic animals than in healthy controls and celastrol early-treated rats (figure 4A). On the contrary, healthy animals and celastrol early-treated animals presented more parallel-lamellae structures. Additionally, arthritic animals showed an increased area occupied by osteocyte lacunae in the total tissue when compared with healthy animals and both celastrol earlytreated and late-treated animals.

\section{Celastrol effect on bone mineral content}

FTIR was performed to assess the composition of cortical and trabecular bone. As shown in figure 5, results demonstrated a decreased mineral and collagen content in trabecular arthritic bone compared with healthy controls (figure 5A,B). Mineral content of trabecular bone was increased in celastrol late-treated group in comparison with arthritic animals (figure 5A). However, the major impact of celastrol was found in cortical bone with increased mineral content in early-treated and late-treated groups compared with arthritic animals (figure 5C). Results also demonstrated a significantly increased carbonate content in early celastrol-treated and late celastrol-treated groups in comparison with the arthritic group (figure 5D).

\section{Celastrol effect on bone mechanical properties}

Bone strength of rat femurs was evaluated using the 3-point bending test (figure 6). There was a reduction in the maximal load that arthritic femurs were able to resist before breaking as compared with healthy controls $(-13.6 \%$, figure $6 \mathrm{~A})$, as well as in the capability to absorb energy (-29.6\%, figure 6C). Early administration of celastrol improved bone strength and maximal load was 
A
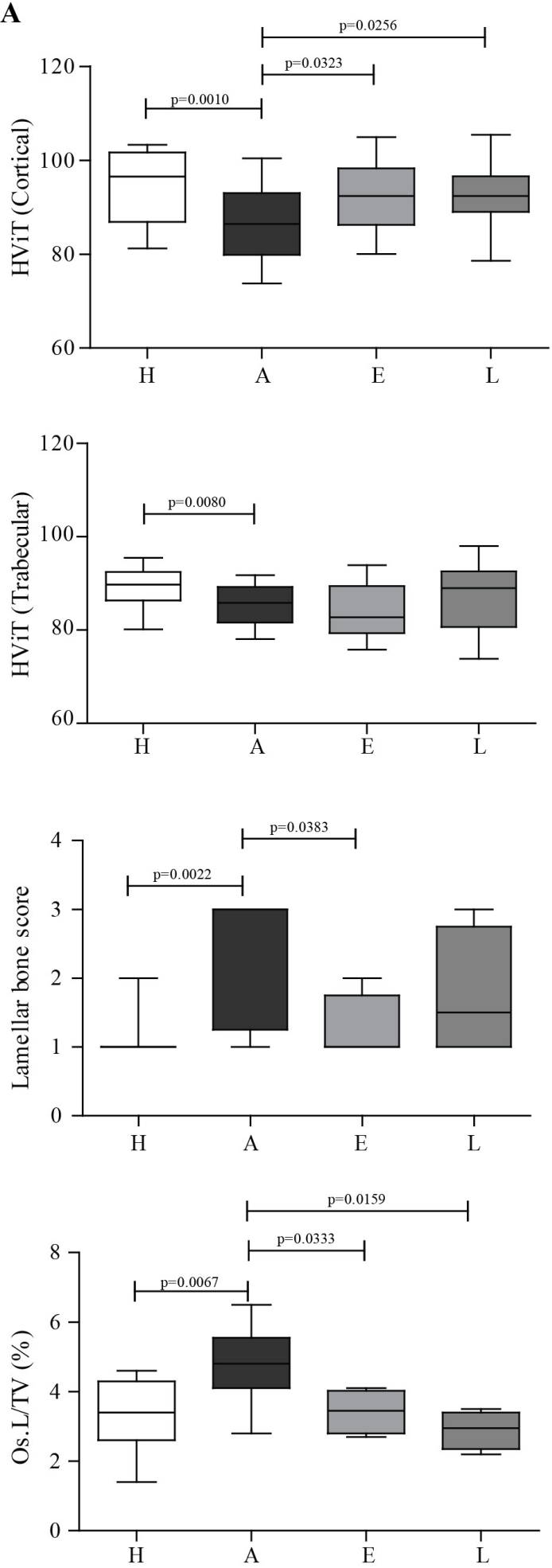

B

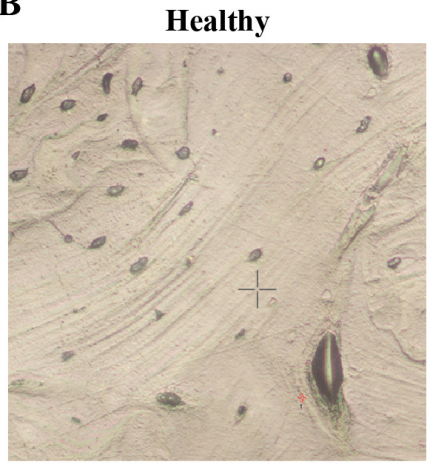

Arthritic

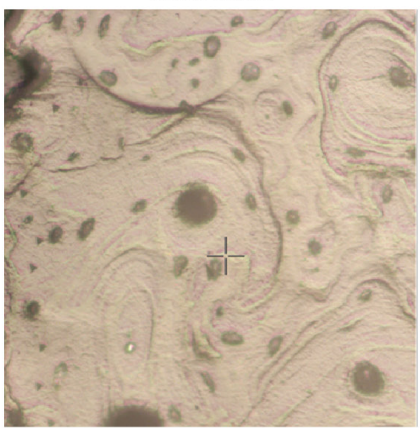

Celastrol early group

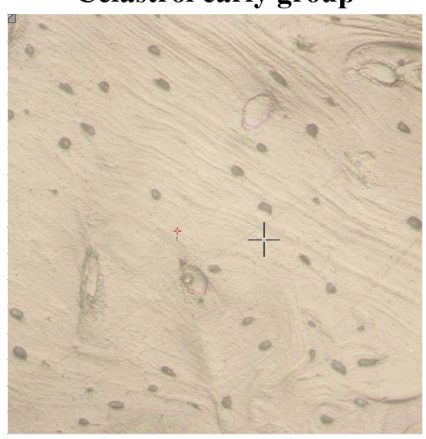

Celastrol late group

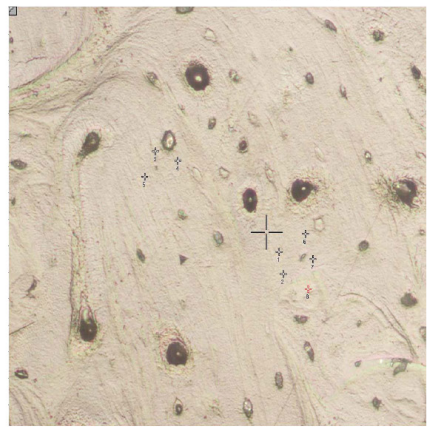

Figure 4 Bone mechanical properties assessed by nanoindentation in rat tibiae on day 22 post disease induction. (A) Results showed decreased cortical and trabecular hardness in the arthritic group when compared with healthy rats. Of notice, rats treated with celastrol showed increased hardness in cortical bone in comparison with untreated arthritic rats. (B) Illustrative topographic images of the histological features observed in the indentation tissue area. Concentric lamellae are identified in secondary osteons, characteristic of arthritic animals. On the contrary, parallel-lamellae are identified in healthy and celastrol early-treated groups. Magnification 20x. Tibiae were collected at the time of sacrifice. Data are expressed as median with minimum and maximum interval. HViT, Vickers hardness; Os.L/TV, osteocyte lacunae per tissue volume; H, healthy; A, arthritic; $E$, celastrol early-treated; L, celastrol late-treated. Healthy, $n=28$; arthritic, $n=21$; celastrol early-treated, $n=14$; and celastrol latetreated, $n=14$. 
A
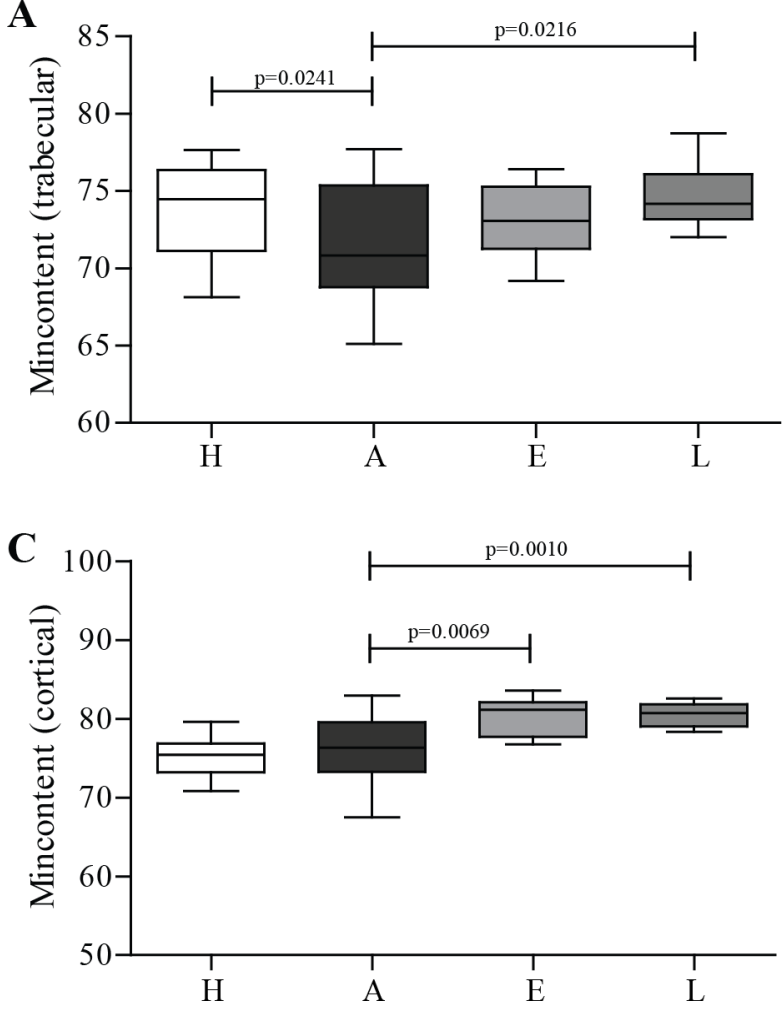

B

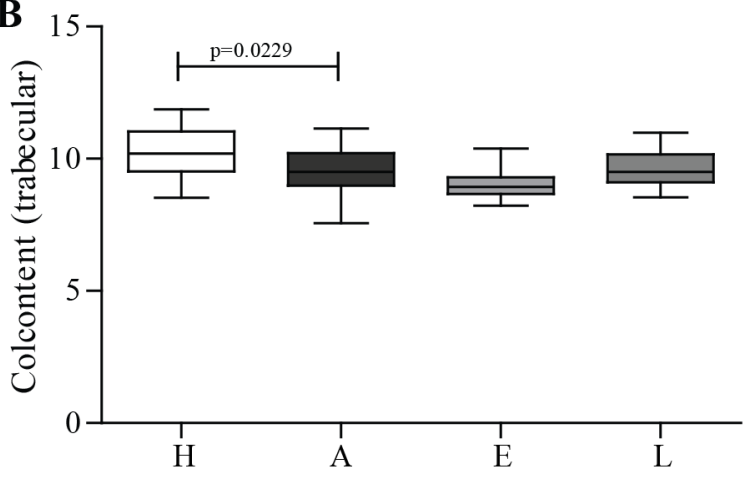

D

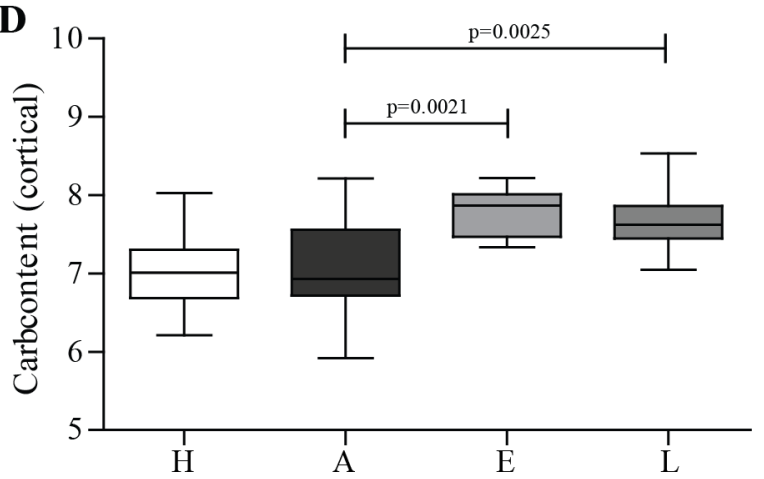

Figure 5 FTIR measurements from cortical and trabecular bone of rat tibia on day 22 post disease induction. FTIR measurements revealed that arthritic rats had mineral and collagen loss in trabecular bone (A and B). Celastrol-treated groups demonstrated increased mineral content of cortical and trabecular bone (A and C), as well as carbonate cortical content (D). Tibiae were collected at the time of sacrifice. Data are expressed as median with minimum and maximum interval. $\mathrm{H}$, healthy; A, arthritic; E, celastrol early-treated; L, celastrol late-treated. Healthy, $n=28$; arthritic, $n=21$; celastrol early-treated, $n=14$; and celastrol late-treated, $n=14$. FTIR, Fourier transform infrared spectroscopy.

increased by $9.4 \%$, when compared with arthritic group. Late celastrol administration was insufficient to correct bone damage, and these animals showed decreased maximal deformation and capability to absorb energy, which were significantly reduced by $-14.7 \%$ (figure $6 \mathrm{~B}$ ) and-18.8\% (figure 6C), respectively. Additionally, arthritic rats had a reduction in the yield displacement compared with healthy controls $(-28,3 \%$, figure $6 \mathrm{D})$. In contrast,
A

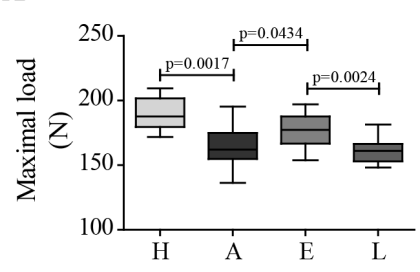

B

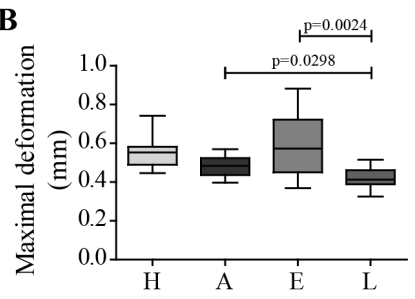

C

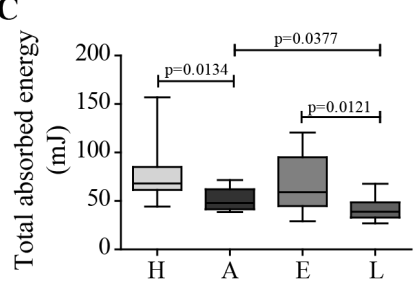

D

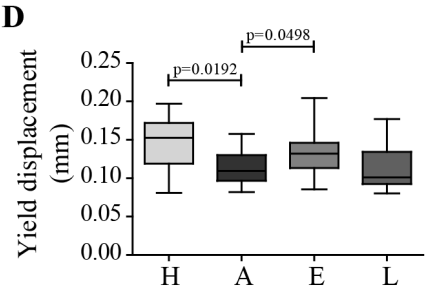

$\mathbf{E}$

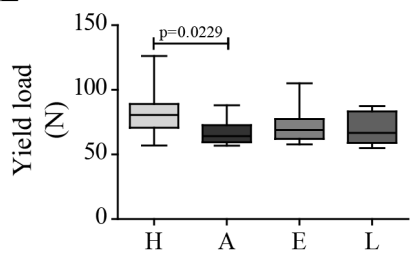

F

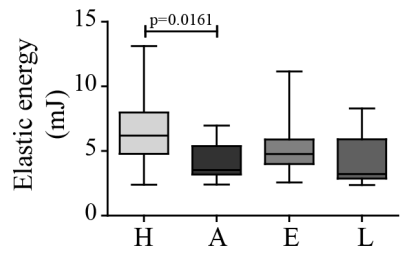

$\mathbf{G}$

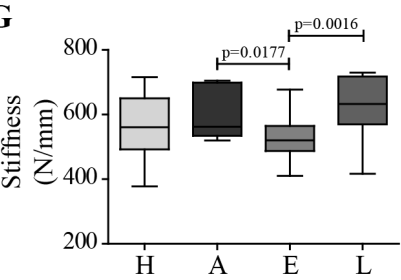

Figure 6 Celastrol effect on arthritic bone mechanical properties. Maximal load (A), maximal deformation (B), total absorbed energy $(C)$, yield displacement $(D)$, yield load $(E)$, elastic energy $(F)$ and stiffness $(G)$ parameters were obtained by 3-point bending. Celastrol early-treated rats have higher levels of yield point displacement and maximum load compared with untreated arthritic rats. Femurs were collected after 22 days of disease induction. Data are expressed as median with minimum and maximum interval. $\mathrm{H}$, healthy; $\mathrm{A}$, arthritic; $\mathrm{E}$, celastrol early-treated; L, celastrol late-treated. Healthy, $n=13$; arthritic, $n=10$; celastrol early-treated, $n=15$; and celastrol late-treated, $n=15$. 
in celastrol early-treated rats, there was an increase in the elastic properties of bone with an augmented yield displacement compared with arthritic rats $(+20.7 \%)$, meaning that a higher elastic deformation of the femur occurred before the appearance of the first microfractures. In addition, mechanical results revealed that there was a significant reduction in the load (figure 6E) and elastic energy at yield point (figure 6F) in arthritic rats compared with healthy controls, only partially corrected in arthritic rats under celastrol treatment since the early phase of the disease $(+7.4 \%$ and $+34.8 \%$ than arthritic rats, respectively). Celastrol early-treated rats also showed a significant reduction in bone stiffness (figure 6G) in comparison to arthritic rats and celastrol late-treated rats $(-7.5 \%$ and $-17.8 \%$, respectively). However, no difference was observed between healthy controls and arthritic rats in this mechanical parameter.

\section{DISCUSSION}

In this study, the AIA rat model was used to assess the bone protective properties of celastrol in vivo, using novel techniques to accurately evaluate bone microarchitecture, mechanics and chemical composition. Here we demonstrated for the first time an inhibition of joint destruction and a reduction of systemic bone degradation after celastrol treatment, translated by the preservation of its structure. This might have implications for RA treatment, as bone disturbances constitute a major issue in this disease. ${ }^{35}$

We have previously shown that celastrol reduces the number of synovial macrophages, ${ }^{21}$ which are critical in osteoclast differentiation. ${ }^{1}$ Most importantly, we had also shown that celastrol inhibits NF-kB activation in vitro, ${ }^{9}$ a crucial step in osteoclastformation and function, upstream activated by the engagement of the receptor activator of NF-kB ligand (RANKL) with RANK. Recently, in vitro findings showed that celastrol inhibits the recruitment of TGF $\beta$-activated kinase (TAK) 1 , an upstream receptor-associated factor of IKB kinase, to RANK and TNF receptors, ${ }^{36}$ inhibiting both RANKL-induced NF-kB activation and the osteoblast-related extracellular signal-regulated kinase (ERK) signalling. ${ }^{19}$ Work from the Moudgil Laboratory has shown, in vitro, that celastrol reduces other osteoclastic mediators besides RANKL, suggesting a shift in bone remodelling in favour of an antiosteoclastic activity. ${ }^{23}$ Finally, it has been reported in bone metastasis and ovariectomy-induced bone loss models that celastrol reduces osteoclast numbers and bone loss and preserves its trabecular architecture, together with an inhibitory effect on osteoblasts' viability and function. ${ }^{19} 37$ By the end of the treatment, AIA rats treated with celastrol had less inflammatory signs and ankle swelling than untreated animals, with no association with drug-induced weight loss nor with increased risk of infections. Also, celastrol administration was not associated with hepatotoxicity, nephrotoxicity nor with lung or spleen disturbances. ${ }^{21}$ In line with this previous evidence, we have shown in this study that celastrol significantly decreases the number of joint tissue osteoclasts and osteoblasts. Importantly, the gold standard treatment for RA, methotrexate, also acts in a similar way, slowing down articular damage by inhibiting osteoclastogenesis ${ }^{38}$ which is coupled to a reduction in osteoblasts proliferation. ${ }^{39}$ The immune-regulatory effects of methotrexate have some overlapping features with celastrol, such as modulation of cytokine secretion ${ }^{40} 41$ and NF-kB activity inhibition, ${ }^{42}$ but also includes reactive oxygen species (ROS) generation leading to cell cycle arrest, apoptosis and changes in monocyte-endothelial adhesion, ${ }^{43}$ inhibition of synovial fibroblasts proliferation and neutrophil chemotaxis, ${ }^{44}$ DNA hypomethylation in blood cells ${ }^{45}$ and defective Treg cell function reversion. ${ }^{46}$ Importantly, despite the lack of a head-to-head comparison analysis, data have recently suggested that an extract of TwHF, containing celastrol, is effective and safe in the treatment of RA and might have an efficacy comparable to the existing conventional synthetic disease-modifying antirheumatic drugs, such as methotrexate. $^{47}$

The reduction in osteoclast and osteoblast numbers is consistent with the significant reduction in serum levels of TRACP-5b and P1NP observed in arthritic rats treated with celastrol, suggesting a reduction in the accelerated bone turnover induced by arthritis.

We have also quantified serum CTX-II. This is a major component of articular cartilage ${ }^{48}$ with a good correlation between serum levels and the severity of cartilage damage. ${ }^{40}$ Celastrol treatment significantly reduced serum CTX-II concentration, suggesting a chondroprotective effect. This protective effect on cartilage could be explained by the inhibition of heat shock protein $90 \beta$ and of NF-kB activation, ${ }^{51}$ combined with the control of inflammation.

Trabecular bone microarchitecture is an important feature of bone quality. ${ }^{52}$ Notably, celastrol treatment significantly improved arthritis-induced trabecular bone degradation and mitigated bone loss. Consistent with this, our results showed that celastrol administration prevented the loss of bone mechanical compliance of femurs in arthritic rats by preserving maximum load and yield displacement. In addition, celastrol-treated arthritic animals also showed positive effects on cortical bone morphology, which is a major factor defining the mechanical properties of bone. Furthermore, celastrol-treated animals had increased bone cortical hardness. This is probably explained by the observation in cortical bone of a higher frequency of parallel-lamellae structures, which are $10 \%$ harder than concentric lamellae. ${ }^{53}$ In fact, concentric lamellae organised in secondary osteons microstructures have been associated with high bone remodelling and decreased hardness in nanoindentation. ${ }^{30} 5354$ Other explanation for the differences related with hardness is that arthritic animals showed an increased number of osteocyte lacunae and of the area occupied by these lacunae in the total tissue. Celastrol early-treated group was able to preserve bone 
matrix, with maintenance of the lacunae area per tissue volume. Osteocytes are responsible for the maintenance of bone homeostasis, and their irregular morphology has been associated with altered ability of the bone to reply to mechanical stimuli leading to significant changes in the structure and mineral density. ${ }^{55}$ In addition, these changes in osteocyte lacunae morphology might be related to osteocyte apoptosis, which could promote bone necrosis, leading to mineral loss and decreased hardness with consequent mechanical weakness. ${ }^{55}$

Finally, FTIR measurements demonstrated that inflammation induces bone mineral and collagen loss in trabecular bone. In contrast, celastrol was able to preserve and even increase mineral content of bone tissue matrix, especially in the late-treated group. FTIR results were also consistent with a decreased bone turnover rate, revealed by significantly increased levels of carbonate content in celastrol-treated groups.

The bone protective effect of celastrol can be mainly attributed to its anti-inflammatory properties, such as inhibition of proinflammatory cytokines, which are upstream of RANKL in the osteoclastogenic pathway and suppression of metalloproteinases (MMPs) via NF-kB inhibition. Celastrol bone-preserving effect has also been demonstrated in cancer models, which is supportive of these data. Cancer and arthritis share common inflammatory pathways such as the NF-kB pathway, which is a molecular target of this small molecule. In addition, in an experimental mice model of secondary osteoporosis, celastrol treatment ameliorated abnormal bone metabolism also via NF-kB and MMPs inhibition. ${ }^{56}$

Despite celastrol efficacy there are still some differences between treated and healthy phenotypes; therefore, it is reasonable to speculate that the extent of bone protection could be even further improved by optimised doses of celastrol or by augmenting treatment duration, which is not possible when using the AIA model.

In conclusion, celastrol halted joint destruction and preserved structural and mechanical bone properties, and thus may serve as a useful therapeutic agent for the treatment of inflammation-induced bone damage.

Contributors Study design: RC, LFM and JEF. Study conduct: RC. Data collection: RC, BV, MAJF and IPL. Data analysis: RC, BV and MAJF. Data interpretation: RC, BV and MAJF. Drafting the manuscript: RC and MAJF. Revising manuscript content: RC, BV, MAJF, IPL, RLT, SS, LFM and JEF. Approving final version of manuscript: RC, BV, MAJF, IPL, RLT, SS, LFM and JEF. RC, BV, MAJF, IPL, RLT, SS, LFM and JEF take responsibility for the integrity of the data analysis.

Competing interests None declared.

Provenance and peer review Not commissioned; externally peer reviewed.

Open Access This is an Open Access article distributed in accordance with the Creative Commons Attribution Non Commercial (CC BY-NC 4.0) license, which permits others to distribute, remix, adapt, build upon this work non-commercially, and license their derivative works on different terms, provided the original work is properly cited and the use is non-commercial. See: http://creativecommons.org/ licenses/by-nc/4.0/

C Article author(s) (or their employer(s) unless otherwise stated in the text of the article) 2017. All rights reserved. No commercial use is permitted unless otherwise expressly granted.
REFERENCES

1. Firestein GS. Evolving concepts of rheumatoid arthritis. Nature 2003;423:356-61.

2. Dequeker J, Geusens P. Osteoporosis and arthritis. Ann Rheum Dis 1990;49:276-80.

3. Schett G, Saag KG, Bijlsma JW. From bone biology to clinical outcome: state of the art and future perspectives. Ann Rheum Dis 2010;69:1415-9.

4. Haugeberg G, Uhlig T, Falch JA, et al. Bone mineral density and frequency of osteoporosis in female patients with rheumatoid arthritis: results from 394 patients in the Oslo County Rheumatoid Arthritis register. Arthritis Rheum 2000;43:522-30.

5. van Staa TP, Geusens P, Bijlsma JW, et al. Clinical assessment of the long-term risk of fracture in patients with rheumatoid arthritis. Arthritis Rheum 2006;54:3104-12.

6. Schett G, Gravallese E. Bone erosion in rheumatoid arthritis: mechanisms, diagnosis and treatment. Nat Rev Rheumatol 2012;8:656-64.

7. Cascão R, Moura RA, Perpétuo I, et al. Identification of a cytokine network sustaining neutrophil and Th17 activation in untreated early rheumatoid arthritis. Arthritis Res Ther 2010;12:R196.

8. Cascão R, Polido-Pereira J, Canhão H, et al. Caspase-1 is active since the early phase of rheumatoid arthritis. Clin Exp Rheumatol 2012;30:144.

9. Cascão R, Vidal B, Raquel H, et al. Effective treatment of rat adjuvant-induced arthritis by celastrol. Autoimmun Rev 2012;11:856-62.

10. Kim DH, Shin EK, Kim YH, et al. Suppression of inflammatory responses by celastrol, a quinone methide triterpenoid isolated from Celastrus regelii. Eur J Clin Invest 2009;39:819-27.

11. Lee JH, Koo TH, Yoon H, et al. Inhibition of NF-kappa B activation through targeting I kappa B kinase by celastrol, a quinone methide triterpenoid. Biochem Pharmacol 2006;72:1311-21.

12. Venkatesha SH, Yu H, Rajaiah R, et al. Celastrus-derived celastrol suppresses autoimmune arthritis by modulating antigeninduced cellular and humoral effector responses. $\mathrm{J} \mathrm{Biol} \mathrm{Chem}$ 2011;286:15138-46.

13. Venkatesha SH, Astry B, Nanjundaiah SM, et al. Suppression of autoimmune arthritis by Celastrus-derived Celastrol through modulation of pro-inflammatory chemokines. Bioorg Med Chem 2012;20:5229-34.

14. Lee JY, Lee BH, Kim ND, et al. Celastrol blocks binding of lipopolysaccharides to a Toll-like receptor4/myeloid differentiation factor2 complex in a thiol-dependent manner. J Ethnopharmacol 2015;172:254-60.

15. Li G, Liu D, Zhang Y, et al. Celastrol inhibits lipopolysaccharidestimulated rheumatoid fibroblast-like synoviocyte invasion through suppression of TLR4/NF-kB-mediated matrix metalloproteinase-9 expression. PLoS One 2013;8:e68905.

16. Li GQ, Zhang Y, Liu D, et al. Celastrol inhibits interleukin-17Astimulated rheumatoid fibroblast-like synoviocyte migration and invasion through suppression of NF-kB-mediated matrix metalloproteinase-9 expression. Int Immunopharmacol 2012:14:422-31.

17. Yu Y, Koehn CD, Yue Y, et al. Celastrol inhibits inflammatory stimuliinduced neutrophil extracellular trap formation. Curr Mol Med 2015;15:401-10.

18. Astry B, Venkatesha SH, Laurence A, et al. Celastrol, a Chinese herbal compound, controls autoimmune inflammation by altering the balance of pathogenic and regulatory T cells in the target organ. Clin Immunol 2015;157:228-38.

19. Idris Al, Krishnan M, Simic P, et al. Small molecule inhibitors of IkappaB kinase signaling inhibit osteoclast formation in vitro and prevent ovariectomy-induced bone loss in vivo. Faseb $\mathrm{J}$ 2010;24:4545-55.

20. Gan K, Xu L, Feng X, et al. Celastrol attenuates bone erosion in collagen-Induced arthritis mice and inhibits osteoclast differentiation and function in RANKL-induced RAW264.7. Int Immunopharmacol 2015;24:239-46.

21. Cascão R, Vidal B, Lopes IP, et al. Decrease of CD68 Synovial Macrophages in Celastrol Treated Arthritic Rats. PLoS One 2015;10:e0142448

22. da Silva JA, Fonseca JE, Graça L, et al. Reinnervation of postarthritic joints in the rat. Clin Exp Rheumatol 1996;14:43-51.

23. Nanjundaiah SM, Venkatesha $\mathrm{SH}, \mathrm{Yu} \mathrm{H}$, et al. Celastrus and its bioactive celastrol protect against bone damage in autoimmune arthritis by modulating osteoimmune cross-talk. J Biol Chem 2012;287:22216-26.

24. Stolina M, Bolon B, Middleton S, et al. The evolving systemic and local biomarker milieu at different stages of disease progression in rat adjuvant-induced arthritis. J Clin Immunol 2009;29:158-74. 
25. Chen DL, Wang DS, Wu WJ, Wj W, et al. Overexpression of paxillin induced by miR-137 suppression promotes tumor progression and metastasis in colorectal cancer. Carcinogenesis 2013;34:803-11.

26. Bouxsein ML, Boyd SK, Christiansen BA, et al. Guidelines for assessment of bone microstructure in rodents using microcomputed tomography. J Bone Miner Res 2010;25:1468-86.

27. Herlin M, Finnilä MA, Zioupos P, et al. New insights to the role of aryl hydrocarbon receptor in bone phenotype and in dioxin-induced modulation of bone microarchitecture and material properties. Toxicol Appl Pharmacol 2013;273:219-26.

28. Zhang R, Gong $\mathrm{H}$, Zhu D, et al. Multi-level femoral morphology and mechanical properties of rats of different ages. Bone 2015;76:76-87.

29. Oliver WC, Pharr GM. An improved technique for determining hardness and elastic modulus using load and displacement sensing indentation experiments. J Mater Res 1992;7:1564-83.

30. Vidal B, Cascão R, Vale AC, et al. Arthritis induces early bone high turnover, structural degradation and mechanical weakness. PLoS One 2015;10:e0117100.

31. Parfitt AM, Drezner MK, Glorieux FH, et al. Bone histomorphometry: standardization of nomenclature, symbols, and units. Report of the ASBMR Histomorphometry Nomenclature Committee. J Bone Miner Res 1987;2:595-610.

32. Isaksson $\mathrm{H}$, Turunen MJ, Rieppo L, et al. Infrared spectroscopy indicates altered bone turnover and remodeling activity in renal osteodystrophy. J Bone Miner Res 2010;25:1360-6.

33. Stolina M, Schett $G$, Dwyer D, et al. RANKL inhibition by osteoprotegerin prevents bone loss without affecting local or systemic inflammation parameters in two rat arthritis models: comparison with anti-TNFalpha or anti-IL-1 therapies. Arthritis Res Ther 2009;11:R187.

34. Stolina M, Adamu S, Ominsky M, et al. RANKL is a marker and mediator of local and systemic bone loss in two rat models of inflammatory arthritis. J Bone Miner Res 2005;20:1756-65.

35. Caetano-Lopes J, Rodrigues A, Lopes A, et al. Rheumatoid arthritis bone fragility is associated with upregulation of IL17 and DKK1 gene expression. Clin Rev Allergy Immunol 2014;47:38-45.

36. Sethi G, Ahn KS, Pandey MK, et al. Celastrol, a novel triterpene, potentiates TNF-induced apoptosis and suppresses invasion of tumor cells by inhibiting NF-kappaB-regulated gene products and TAK1-mediated NF-kappaB activation. Blood 2007;109:2727-35.

37. Idris $\mathrm{Al}$, Libouban $\mathrm{H}$, Nyangoga $\mathrm{H}$, et al. Pharmacologic inhibitors of IkappaB kinase suppress growth and migration of mammary carcinosarcoma cells in vitro and prevent osteolytic bone metastasis in vivo. Mol Cancer Ther 2009;8:2339-47.

38. Kanagawa $\mathrm{H}$, Masuyama $\mathrm{R}$, Morita $\mathrm{M}$, et al. Methotrexate inhibits osteoclastogenesis by decreasing RANKL-induced calcium influx into osteoclast progenitors. J Bone Miner Metab 2016;34.

39. Annussek T, Kleinheinz J, Thomas S, et al. Short time administration of antirheumatic drugs - methotrexate as a strong inhibitor of osteoblast's proliferation in vitro. Head Face Med 2012;8:26.

40. Sperling RI, Benincaso Al, Anderson RJ, et al. Acute and chronic suppression of leukotriene B4 synthesis ex vivo in neutrophils from patients with rheumatoid arthritis beginning treatment with methotrexate. Arthritis Rheum 1992;35:376-84.

41. Williams AS, Jones SG, Goodfellow RM, et al. Interleukin-1beta (IL-1beta) inhibition: a possible mechanism for the anti-inflammatory potency of liposomally conjugated methotrexate formulations in arthritis. Br J Pharmacol 1999;128:234-40.

42. Spurlock CF. 3rd, Tossberg JT, Matlock BK, et al. Methotrexate inhibits NF-kappaB activity via long intergenic (noncoding) RNA-p21 induction. Arthritis Rheumatol 2014;66:2947-57.

43. Phillips DC, Woollard KJ, Griffiths HR. The anti-inflammatory actions of methotrexate are critically dependent upon the production of reactive oxygen species. $\mathrm{Br} J$ Pharmacol 2003;138:501-11.

44. Kremer JM. The mechanism of action of methotrexate in rheumatoid arthritis: the search continues. J Rheumatol 1994;21:1-5.

45. de Andres MC, Perez-Pampin E, Calaza M, et al. Assessment of global DNA methylation in peripheral blood cell subpopulations of early rheumatoid arthritis before and after methotrexate. Arthritis Res Ther 2015;17:233.

46. Cribbs AP, Kennedy A, Penn H, et al. Methotrexate Restores Regulatory $\mathrm{T}$ Cell Function Through Demethylation of the FoxP3 Upstream Enhancer in Patients With Rheumatoid Arthritis. Arthritis Rheumatol 2015;67:1182-92.

47. Wang HL, Jiang $Q$, Feng $X H$, et al. Tripterygium wilfordii Hook $F$ versus conventional synthetic disease-modifying anti-rheumatic drugs as monotherapy for rheumatoid arthritis: a systematic review and network meta-analysis. BMC Complement Altern Med 2016;16:215.

48. Elsaid KA, Chichester CO. Review: Collagen markers in early arthritic diseases. Clin Chim Acta 2006;365(1-2):68-77.

49. Oestergaard S, Chouinard L, Doyle N, et al. The utility of measuring C-terminal telopeptides of collagen type II (CTX-II) in serum and synovial fluid samples for estimation of articular cartilage status in experimental models of destructive joint diseases. Osteoarthritis Cartilage 2006;14:670-9.

50. Oestergaard S, Chouinard L, Doyle N, et al. Early elevation in circulating levels of $\mathrm{C}$-telopeptides of type II collagen predicts structural damage in articular cartilage in the rodent model of collagen-induced arthritis. Arthritis Rheum 2006;54:2886-90.

51. Ding QH, Cheng Y, Chen WP, et al. Celastrol, an inhibitor of heat shock protein $90 \beta$ potently suppresses the expression of matrix metalloproteinases, inducible nitric oxide synthase and cyclooxygenase-2 in primary human osteoarthritic chondrocytes. Eur J Pharmacol 2013;708(1-3):1-7.

52. Dempster DW. The contribution of trabecular architecture to cancellous bone quality. J Bone Miner Res 2000;15:20-3.

53. Dall'Ara E, Ohman C, Baleani M, et al. Reduced tissue hardness of trabecular bone is associated with severe osteoarthritis. J Biomech 2011;44:1593-8.

54. Bailey AJ, Mansell JP, Sims TJ, et al. Biochemical and mechanical properties of subchondral bone in osteoarthritis. Biorheology 2004:41(3-4):349-58.

55. Jaiprakash A, Prasadam I, Feng JQ, et al. Phenotypic characterization of osteoarthritic osteocytes from the sclerotic zones: a possible pathological role in subchondral bone sclerosis. Int J Biol Sci 2012;8:406-17.

56. Liu X, Cai F, Zhang Y, et al. Celastrol, an NF-кB inhibitor, ameliorates hypercalciuria and articular cartilage lesions in a mouse model of secondary osteoporosis. J Pharmacol Sci 2016;130:204-11. 